\title{
Operation Efficiency Assessment Model of Route Network in Terminal Area
}

\author{
Zhang Zhao-ning ${ }^{1}$, Zhao Zhao-na', Liu Zhen
}

\begin{abstract}
Terminal area is an important part of airspace system. Its operation efficiency is related to the safety, order and efficiency of the entire air traffic. So the assessment of the terminal route network operation efficiency is needed. Considering the aspects of route segment capacity, flight flow, workload and operation stability, the paper analyzes the influent factors on the operation efficiency of terminal area route network, constructs the calculation parameters of operating efficiency, and establishes the calculation model of operating efficiency of the route segment. An assessment model for the operation efficiency of terminal area route network based on flight demand is built using comprehensive weighted method. Finally, the feasibility of the assessment model is verified by analyzing a case. The model can be used to assess not only the operation efficiency of a single route segment based on different flight demands in the terminal area, but also the real-time efficiency of the terminal area route network.
\end{abstract}

KEYWORDS: Terminal area, Route network, Operation efficiency, Efficiency assessment, Operation stability, Flight flow.

\section{INTRODUCTION}

With the rapid development of civil aviation and the increase of air traffic flow, airspace users' demand for airspace resources is increasing. To ensure safety, efficiency, and orderly operation of air traffic, a sustainable planning of route network and a reasonable dynamic air traffic flow management strategy are needed. Terminal area airspace, as a bottleneck of the whole route network system, is extremely easy to cause the air traffic congestion which seriously affects the safety and effectiveness of air traffic. In order to use terminal area resource flexibly and improve the operation efficiency of route network in terminal area, the research on the operation efficiency assessment of the terminal area route network system is very necessary.

At present, little research is done on route network operation efficiency. For the operation efficiency of airspace system, Federal Aviation Administration assesses the efficiency of airspace system from the aspects of flexibility, predictability, access, delay and so on to ensure the safe, efficient, and orderly movement of air transportation (Federal Aviation Administration 1997). EUROCONTROL built the Performance Review Committee, which proposes nominees to form a Key Performance Indicator Drafting Group (KPIDG) that put forward the indicators of safety, delay, cost effectiveness, flight efficiency predictability, environment equity, access, flexibility and reliability Performance Review Unit (2001). For the terminal area, Rakas and Hansen (2002) developed a methodology for modeling the terminal airspace service availability and system effectiveness; the methodology distinguished the equipment availability, weather availability and service availability, defined each measure, and considered weather and equipment availability jointly. Bloem and Kopardekar (2008) evaluated 
the utilization of a large area with multiple sectors based on Monitor Alert Parameter (MAP) by using the sector-hourly utilization metrics. Zhang et al. (2013) established a general index system which includes density of air traffic flow, safety performance, efficiency performance and workload of air traffic controller, and presented a classification evaluation method by means of artificial intelligence on the basis of back propagation network model. Xu and Yu (2003) proposed conflict detection method based on the flight route segment analysis according to the relationship between radar track and route. In addition, Li et al. (2009a) applied flow theory to the research of route network structure stability. Li et al. (2009b) used the following theory to study the stability of terminal area and proposed the terminal area capacity assessment method based on the flow stability.

For road traffic, the scholars have made achievements on the research of reliability and operation efficiency of road network. Asakura (1996) proposed the travel time reliability and considered the recession of network capacity due to deterioration of road conditions, as well as regarded that travel time reliability is a function of the ratio between the operating time in the blocked state and the unblocked operating time. Latora and Marchiori $(2002,2004)$ proposed to use the shortest path in the network to measure efficiency and applied it to the analysis and evaluation of various networks, but this method analyses efficiency of network only from the travel distance and it ignored some important factors such as flow and others which affected the efficiency of the network. Zhu et al. (2000) studied the reliability, sensitivity and efficiency of road sections using the generation method that is suitable for large-scale network. Jenelius et al. (2006) proposed a method to measure the important degree of road sections. Qin and Shi (2008) put forward a method to measure the traffic network efficiency according to the important degree of the node and link, and it could reflect the influence of the traffic demand, traffic network flow, travel costs and other factors on efficiency of the network comprehensively. Hou and Jiang (2000) studied the survival and effective aspects in the city road network and proposed the function relation based on the path and intersection reliability. Zhan et al. (2010) formulated the aircraft arrival sequencing and scheduling (ASS) problem in the form of a permutation problem and proposed a new solution framework that makes the first attempt at using an ant colony system (ACS) algorithm based on the receding horizon control (RHC) to solve it. D'Ariano et al. (2012) presented the Terminal Manoeuvring Area-Flow Management (TMA-FM) models and algorithms for a busy terminal area and evaluated the retiming and rerouting algorithms on practical size instances from the Fiumicino (FCO) airport. Samà et al. (2014) established the model of air traffic flows in the Terminal Control Area (TCA) based on alternative graphs to solve the real-time problem of aircraft scheduling and routing in TCA. All these ideas and methods provide reference for the index of route segment operation impedance and operation stability to build the assessment model of the operational efficiency in terminal system area. Samà et al. (2013) introduced a rolling horizon framework to manage busy traffic situations with a large number of delayed aircraft. As scheduling algorithms, the paper compared a Branch and Bound (BB) algorithm with a First Come First Served (FCFS) rule that they used as a surrogate for the dispatchers behavior. D'Ariano et al. (2015) enriched the existing models by including new formulations of relevant practical constraints compared with the current literature based on job shop scheduling applied to solve the Aircraft Scheduling Problem (ASP). The paper introduced and analyzed three alternative ASP formulations, in which the objective function is the minimization of delay propagation with respect to the off-line timetable.

In this study, in order to describe the effect of the resource usage, controller workload and air traffic flow stability on the running status of terminal area under different flight flows, the concept of terminal area route network operation efficiency is proposed. This paper analyzes the influence factors of terminal area route network operation efficiency and establishes the calculation index of "route segment traffic efficiency", "workload influence degree" and "route segment operation stability". An assessment model on the operation efficiency of terminal area route network based on flight demand is built. Through the case analysis, the feasibility of the assessment model is verified. We can get that the route network operation stability is fluctuating with the change of air traffic flow, operating efficiency increases first and then decreases with the increase of air traffic flow. The model can be used to assess not only the operation efficiency of a single route segment in the terminal area, but also the real-time efficiency of the terminal area route network. The model will benefit the airspace management and serve as a theoretical basis for scientific exploration of airspace system. In addition, the model can provide reference for the dynamic distribution of traffic, according to the different operation efficiencies. 


\section{ESTABLISHMENT OF TERMINAL AREA ROUTE NETWORK SYSTEM}

The network is expressed by graph in mathematics and the complex network can be accurately described with the language and symbol of graph theory. The graph in graph theory is composed of the number of given points and the lines connecting the two points. The graphic is usually used to describe a specific relationship between certain things, which uses dots to represent things and uses the line connecting the two points to express this relationship between two things. A specific network graph can be abstracted as a graph consisted with a node set $V$ and an edge set $E$. Each side in $E$ corresponds to a pair of nodes in $V$ (Watts and Strogatz 1998; Wilson 1986).

Terminal area airspace system as an important subsystem of the whole airspace system, to some extent, the situation of terminal area is the most complex subsystem. The terminal route network is a structural system that is connected by the route in terminal area airspace according to a certain way. The route network in terminal area is composed of the airport, route, air corridor, aircraft and other elements. Airports, routes and air corridors constitute the space distribution of terminal area network. And the planes complete the arrival or departure through the route and air corridor in the terminal area. The specific operation is that all arrival flights are separated from the flight route and join the airport route for flight, finally through a different approach to the runway line for landing. And all departure flights takeoff from the airport and, along the departure route in terminal area, are finally away from terminal area through corridor and join the route. In this process, it is necessary to make full use of the terminal area resources to meet the demand of arrival and departure flights, to assure the flight safety, efficiency and orderly operation under the command of controller.

The terminal area route network is connected by the routes in a certain way. It is a 3-D network structure that is made up of beacons, airports, location reports and the route segments which connect these waypoints. By projecting the 3-D network onto a 2-D plane, the terminal area route network is defined as a directed graph $G(V, R)$, according to the graph theory. And beacons, airports and location reports can be route network nodes. The node set is $V=\left\{v_{1}, v_{2}, v_{3}, \ldots, v_{\mathrm{n}}\right\}$. The route segments which connect these waypoints are the edge of the network. The route segment set is $R=\left\{r_{1}, r_{2}, r_{3}, \ldots, r_{\mathrm{m}}\right\}$. Each route segment $r$ corresponds to the node $\left(v_{i}, v_{j}\right)$, where $i=1,2, \ldots, m$ and $j=1,2, \ldots, n, r \in R$.
To express the relationship between route segment and node, $G=\left\{g_{i j}\right\}$ is $m \times n$ order incidence matrix between route segment and node in the terminal area route network $G(V, R)$. The element $g_{i j}$ in the matrix is the $0-1$ variable, which is $g_{i j}\left\{\begin{array}{l}1 \\ 0\end{array} g_{i j}=1\right.$ indicates that the waypoint $v_{j}$ is one of the endpoints of route segment $r_{i} ; g_{i j}=0$ indicates that the waypoint $v_{j}$ is not one of the endpoints of route segment $r_{i}$.

\section{TERMINAL AREA ROUTE NETWORK EFFICIENCY AND INFLUENCE FACTORS}

System efficiency can be defined as the degree of realizing the function of the system with the effective utilization of the system resources during the system operation process Jenelius et al. (2006). In the process of the terminal system operation, we need to make full use of resources to complete the terminal area flight approach and departure and to guarantee safety and order of the traffic flow, under the command of the controller. Therefore, the operation efficiency of terminal area route network can be defined as the degree of meeting a certain flight demand with efficient utilization of terminal area existing resources under the command of the controller, while ensuring the flight safety and orderly operation.

The terminal area is a limited airspace, whose operation is closely related to the route structure, control workload, flight flow as well as the external variable factors (e.g. weather conditions, air traffic control system and technical conditions). The terminal area route network operation efficiency is affected by many factors that are subdivided into the following categories:

- Route segment capacity. Route segment is the minimum constituent unit of the terminal area and its capacity is the ultimate reflection of existing resources in the terminal area. The utilization of route segment capacity impacts on the operation efficiency.

- Flight flow. The determination of the terminal area operation is to make the flight flow in an orderly and stable state. With the flight flow increase, air traffic complexity and control workload will increase at the same time. If flight flow is close to the airspace capacity, it may cause traffic congestion and lead to unstable operation of the route network system. As a result, this will affect the entire terminal operation efficiency and safety. Therefore, flight flow is a key factor of operation efficiency. 
- Controller and workload. At present, almost countries mainly use the Air Traffic Control (ATC) route operation mode. Controllers have decision-making power on the whole airspace system traffic control. Pilots comply with controllers' instruction. The goal of controllers' work is to ensure air traffic safety and maximize the use of limited airspace resources. So the controllers will affect the operation efficiency of the route network system in terminal area. Aircraft flight will form the objective task needs for controllers and they must bear the physical and mental pressure to meet these needs. The pressure can be transformed into time consumption. Therefore, the length of time consumption can be expressed by controllers' workload. Controllers' workload will affect the airspace capacity and effective operation. The size of the workload is affected by the complexity of airspace structure, air traffic density and system availability. So the controllers' workload is an important factor of operation efficiency.

- Segment time of flight. The segment time of flight reflects the stability and service capabilities of the terminal, which can also affect the cost of air transport and the efficiency of the entire airspace system. If the flight time in the segment is shorter, it means that this segment operation is stably. If the flight time is longer, it indicates that the operation impedance is relatively big and the segment operation is not stably, which will bring low cost efficiency. Thus, segment time has significant effect on the route network of the terminal area.

- The ATC guarantees facilities. It mainly refers to the distribution, degree of automation and operation reliability of communication, navigation and surveillance equipment. If the precision of ATC facilities changes, ATC service ability to ensure flight will change. In addition, the change of all random factors can also lead to the change of workload, and then it affects the efficiency of route network in terminal area.

- Meteorological conditions. Different weather conditions have different requirements and restrictions on the operation of aircraft, which will affect the controller's workload, as well as the running stability in terminal area, and will ultimately affect the efficiency of terminal area route network. In addition, the weather change can also lead to the change in workload, and then it affects the efficiency of route network in terminal area.

- Some other physical or technical factors. It is mainly reflected on the navigation precision of the equipment and aircraft performance such as rate of climb, the final approach speed etc. To sum up, the determinants mainly include flight flow, controller's workload and segment running time. Furthermore terminal area route network operation efficiency is affected by some external factors such as weather, ATC equipment and precision.

\section{THE ASSESSMENT MODEL OF TERMINAL AREA ROUTE NETWORK OPERATION EFFICIENCY}

Considering the impact of traffic flow characteristics on the air traffic operation efficiency, an assessment model for the operation efficiency of terminal area route network based on flight demand is built. The process of the whole model is shown in Fig. 1.

From Fig. 1, the internal and external factors of the operation efficiency of route network in terminal area are firstly analyzed. Then the main factors are selected taking the flight flow as the starting point. Making certain assumptions, the segment operation efficiency evaluation model is constructed through the establishment of three calculation indexes. Finally, by using weighted method, an assessment model for the operation efficiency of terminal area route network based on flight demand is built.

In the full consideration of air traffic flow influence on the route network operation efficiency, this study builds an assessment model for the operation efficiency of terminal area route network based on flight demand. The basic assumptions of the model are:

- The route is the one-way route, so the route flow mentioned is the one-way flow.

- The controllers' workload regarding the command of an aircraft is certainly in a particular route segment.

\section{ESTABLISHMENT OF THE ASSESSMENT MODEL}

Terminal area route network operation makes full use of the terminal area resources to complete the task of arrival and 


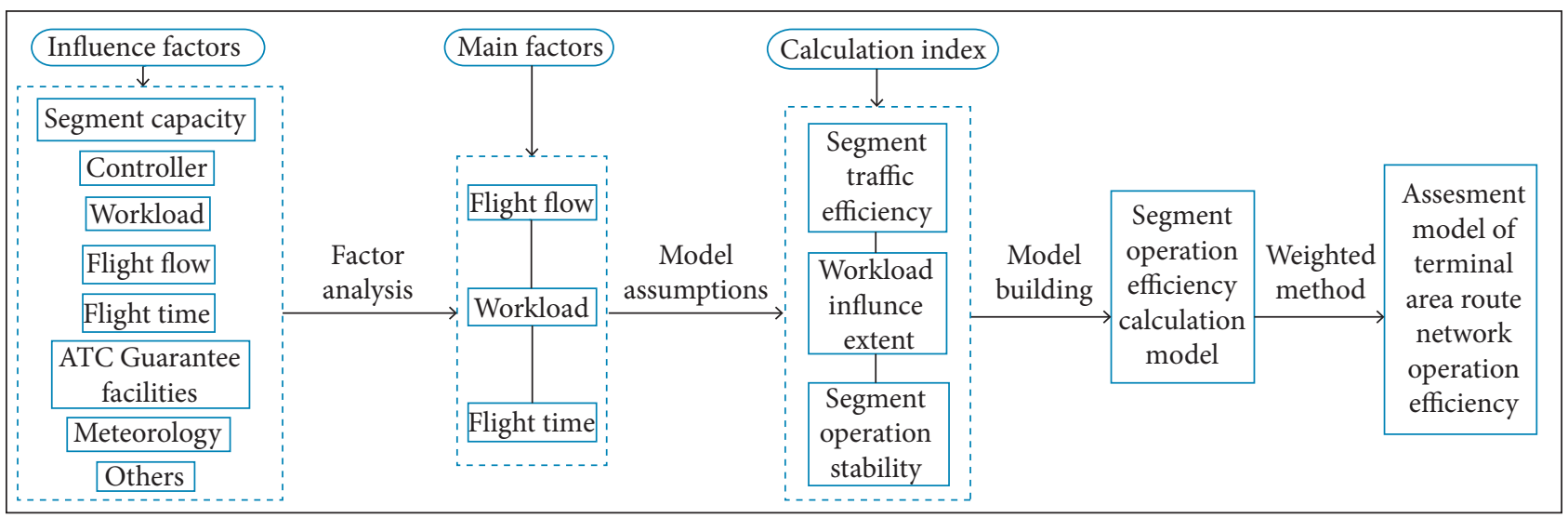

Figure 1. The process of building the assessment model.

departure under the preconditions of guaranteeing security, order, and efficient operation of flight. According to the influencing factors and the operation in the terminal area, we can know that resource utilization in the terminal area is reflected on its airspace capacity utilization rate. Safe operation needs a controller, and it is closely related with the workload. The order and efficiency of the operation can be reflected on the operation stability. So this paper proposes three calculation parameters which are "segment traffic efficiency", "control workload influence extent" and "segment operation stability", based on the flight flow, segment capacity, segment operating stability and controller's workload. So the segment operating efficiency model is established as follows:

$$
e=g(q, c, w, r)
$$

where:

$q$ is the flight flow; $c$ is the route segment capacity; $w$ is the workload of a route segment; and $r$ is the route segment operation stability.

\section{Route Segment Traffic Efficiency}

Route segment traffic efficiency is defined as the ratio of the actual flight flow and the capacity, which can reflect the utilization of the route segment capacity under the normal condition of a certain weather, airspace structure and technique.

To calculate the route segment traffic efficiency, we can count each route segment in the assessed period according to the flying radar data and flight plan. The flight flow is denoted as $q$. Then, when determining the route segment capacity $c$, the average speed through the segment $i$ in the evaluation time period $t$ is $v_{i}$, the length of route segment is $l_{i}$, the minimum safety separation in the segment is $S$, and the separation margin $\Delta S$ is proposed concerning the precision of navigation, communication delay, climate change and other influence on the route capacity. The maximum capacity of route network segment can be expressed as:

$$
c=\frac{l_{i}+v_{i} t}{S+\Delta S}
$$

The route segment traffic efficiency is denoted as:

$$
e_{\text {tra }}=q / c
$$

\section{Workload Influence Extent}

Route segment workload refers to the consumption of working time based on the control instruction during the flight operation in the assessed period. Effect degree of route segment workload is the influence degree of a certain route segment workload on the route segment operation efficiency.

Control workload is closely linked to sorties of regional flights. With the increase of air traffic flow, control workload also increases and the impact on route segment operation efficiency is negative; $f_{i}$ is the workload when the controller commands a plane in the assessed route segment, so the total workload of the segment is:

$$
w=\sum f_{i} q_{i}
$$

where:

$q_{i}$ is the evaluation period, the actual flight flow of segment $i$. The route segment workload influence degree is expressed as:

$$
e_{w o l}=1-w / t
$$




\section{Route Segment Operation Stability}

The reliability of road network goes gradually from abstract road network to the network with traffic flow, because of the study on the road network travel time reliability. It is no longer considered physical structure network simply, taking into account the mutual influence of travel demand and the capacity of road network, as well as travel behavior of road user. Travel time reliability is a very important measure of network reliability index, which describes the probability that the transportation can be finished in a time range. Air transport is in pursuit of high speed and high efficiency. Users and management of terminal route network attach great importance to the economic operation. So the flight time of aircraft in the terminal area is more concerned. And the operation stability has obvious effects on the segment efficiency of route network. Therefore, the research methods of the road network travel time reliability are worth learning to discuss the operation stability of route segment.

Reliability of travel time in the road network mainly considers the impact of traffic demand and the capacity change of road network on its reliability. But flight flow at different moments in the route network is changing, and capacity is affected by the aircraft type, the precision of navigation, communication delay and weather conditions as well as other variable factors, so the actual capacity of route is within certain changes randomly. Aircraft flying in the terminal area is ordered optimized. The aircrafts are affected by each other and the behind aircraft is interfered by the front aircraft. The route operation in the terminal is similar to the road operation through analysis. The research methods of road travel time reliability are learned to establish a terminal area route network operation stability model. This can no longer simply consider the physical structure of route network, but takes the mutual influence of flight demand and route network capacity, as well as the impact of workload, into account.

Route segment operation stability is defined as the probability of meeting the flight demand within certain operation impedance Ming (2006).

Route segment operation impedance is the obstruction impact on flight flow in the route segment. Route segment operation impedance is a function of flight flow. With the increase of air traffic flow, segment presents congestion or fluctuating phenomenon, and this will result in the increasing of operating impedance in section. But the actual increasing change of operating impedance has no mature model to calculate it in the civil aviation field. USA Federal Highway
Administration summarized the relationship between travel time and traffic flow of road and proposed the road impedance formula, which is the Bureau of Public Roads (BPR) function (Sheffi 1985). The operation of route network in the terminal area is similar to that of road network. Draw on the experience of BPR function, route segment operation impedance can be computed as follows:

$$
t_{a}=t_{0}\left[1+\alpha\left(\frac{q}{c_{a}}\right)^{\beta}\right]
$$

where:

$t_{0}$ is the route segment zero flow impedance and represents the time that a plane in free flight requires the time when the flight flow is zero. It can be calculated based on the average flight speed, expressed as $t_{0}=l_{\mathrm{i}} / \mathrm{v}_{\mathrm{i}} ; c_{a}$ is the actual capacity of the segment and it presents randomness with the change of route network operating environment; $\alpha$ and $\beta$ are the model parameters, $q / c_{a} \leq 1$, because the flight flow is not allowed to exceed the capacity in route. According to the improved BPR function Wang et al. (2006), we can get $\alpha=0.5668$ and $\beta=1.4431$.

Because the aircraft type, the precision of navigation, communication delay and weather conditions as well as human factor are changing, so the actual travel ability of segment is random. Based on the actual operating situation, it can be assumed that route segment capacity follows the uniform distribution. The probability density function is as follows:

$$
f_{c}(\mathrm{x})=\left\{\begin{array}{l}
\frac{1}{\bar{c}(1-\theta)}, \overline{\theta c}<\mathrm{x}<\bar{c} \\
0, x \leq \theta \bar{c}, x \geq \bar{c}
\end{array}\right.
$$

where:

$\mathrm{x}$ and $\mathrm{x}$ are the upper and lower density function, respectively; $\theta(0 \leq \theta \leq 1)$ is the use rate of route segment ability, when the weather, equipment and other condition are changing.

Assuming that the route segment capacity is independent of air traffic flow, the mean and variance of route segment operation impedance are obtained according to Eq. 7, which can measure the impact of operation impedance on the whole operation progress. The mean and variance are calculated as follows:

$$
E\left(t_{a}\right)=t_{0}+t_{a} E\left(\frac{q}{c_{a}}\right)^{\beta}=t_{0}+\alpha t_{0} q^{\beta} \frac{1-\theta^{1-\beta}}{\bar{c}(1-\theta)(1-\beta)}
$$




$$
\begin{aligned}
D\left(t_{a}\right)= & \alpha^{2} t_{0}{ }^{2} q^{2 \beta} D\left(\frac{1}{c_{a}}\right)^{\beta}= \\
& \alpha^{2} t_{0}{ }^{2} q^{2 \beta}\left\{\frac{1-\theta^{1-2 \beta}}{\bar{c}^{2 \beta}(1-\theta)(1-2 \beta)}-\left[\frac{1-\theta^{1-\beta}}{\bar{c}(1-\theta)(1-\beta)}\right]^{2}\right\}
\end{aligned}
$$

According to the research of literature (Wilson 1986), the route segment operation impedance follows the normal distribution. Route segment stability refers to the probability of completing the flight demand under a certain route segment operation impedance.

The route segment operation impedance is expressed as:

$$
e_{s t a}=P\left\{t_{a} \leq t\right\}=P\left\{t_{a} \leq \psi t_{0}\right\}
$$

where:

$\Psi=E\left(t_{0}\right) / t_{0}+\varepsilon ; \Psi$ is the congestion coefficient of route segment, and $\varepsilon$ is the parameter that describes the operation impedance deviation from the minimum average running time. In order to ensure the normal operation of flight, we take $\varepsilon=0.015$.

\section{Route Segment Operation Efficiency}

Terminal area route network operation efficiency is heavily influenced by air traffic flow. During the calculation of route network operation efficiency, we take controller's workload, air traffic flow and the route segment operation stability into account. The significance of route segment operation efficiency is that air traffic not only meets a certain transport demand but also ensures the orderly, safe and smooth operation.

The route segment operation efficiency is expressed as follows:

$$
e_{\mathrm{r}}=e_{t r a} \cdot e_{w o l} \cdot e_{s t a}=\frac{q}{c} \cdot\left(1-\frac{w}{t}\right) \cdot P\left\{t_{a} \leq \psi t_{0}\right\}
$$

\section{Terminal Area Route Network Operation Efficiency}

Each route segment operation is relatively independent at the same period in the entire route network. They jointly influence the route network system terminal operation. We can adopt comprehensive weighted method to calculate the terminal network operation efficiency. Considering the characteristics of traffic flow (Wilson 1986), each route segment of the transport turnover is different and the impact on the terminal route network operation efficiency is different, so the route segment turnover can be a calculation weight.

The terminal area route network operation efficiency is calculated as follows:

$$
\mathrm{E}_{\mathrm{t}}=\sum_{i=1}^{m_{0}} e_{r i} \frac{q_{i} l_{i}}{\mathrm{~g}(q l)}
$$

where:

$e_{r i}$ is the operation efficiency of route segment $i ; g(q l)$ is the traffic turnover of terminal, and its value is equal to the sum of each route segment turnover in the assessed terminal area. It is calculated as:

$$
g(q l)=\sum_{i=1}^{m_{0}} q_{i} l_{i}
$$

where:

$m_{0}$ is the total quantity of route segments and $i$ in the terminal area.

\section{NUMERICAL EXAMPLE}

We select an approach route network in the south of a terminal area to calculate and analyze the efficiency of an hour. According to the graph theory, the system diagram of route network is built. The route network node set is $V=$ \{AND, IDINK, DADAT, JTN $\}$ and the route network section set is $R=\{$ AND - DADAT, AND - IDINK, DADAT - JTN, IDINK - JTN $\}$. Figure 2 shows the system diagram of route network.

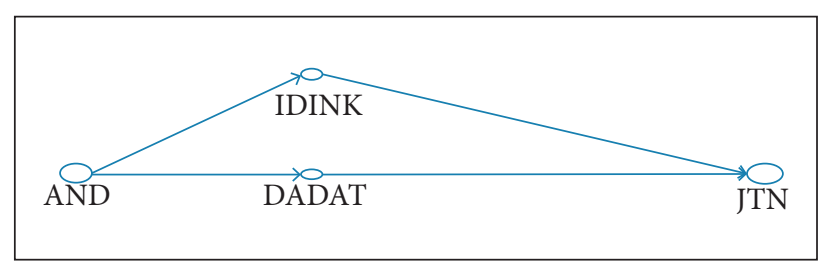

Figure 2. System diagram of route network.

We can get that the minimum safety separation $S=10 \mathrm{~km}$ based on the flight rules, the separation margin $\Delta S=5 \mathrm{~km}$ in consideration of the precision of navigation, communication delay, climate change and other random influence factors. The length of segment and the speed of aircraft can be obtained through collecting statistic. The controllers' workload in the command of an approaching plane is $101.2 \mathrm{~s}$ by analyzing the 
selected data of radar and voice. Table 1 shows the capacity and the length of route segment.

\section{THE NUMERICAL EXAMPLE BASED ON FLIGHT DEMAND}

Concerning the calculation of route segment stability, Fig. 3 shows the operation stability of the route network in the terminal area based on flight demand. Regarding the assessment model, Fig. 4 shows the operation efficiency of the route network of the terminal area based on flight demand.

According to the results based on the assessment model, we can get that the operation of segment is steady and ordered when the flow is small. And the reliability of each route segment decreases with the increase of the air traffic flow. When the traffic flow is close to the maximum segment capacity, the operation stability degree is close to 0.6 . In addition, according to the four segment operation stabilities of AND - DADAT, AND - IDINK, DADAT - JTN and IDINK - JTN, we can know that when the flight flow is given, the bigger the capacity of the route segment, the more stable the air traffic flow system.

The value of operational efficiency of route network in the terminal can be assessed by the model. From Fig. 4, we know that, with the increase of air traffic flow in terminal area, the operation efficiency will increase firstly. The reason is that the air traffic flow increases, the workload increases, and the route

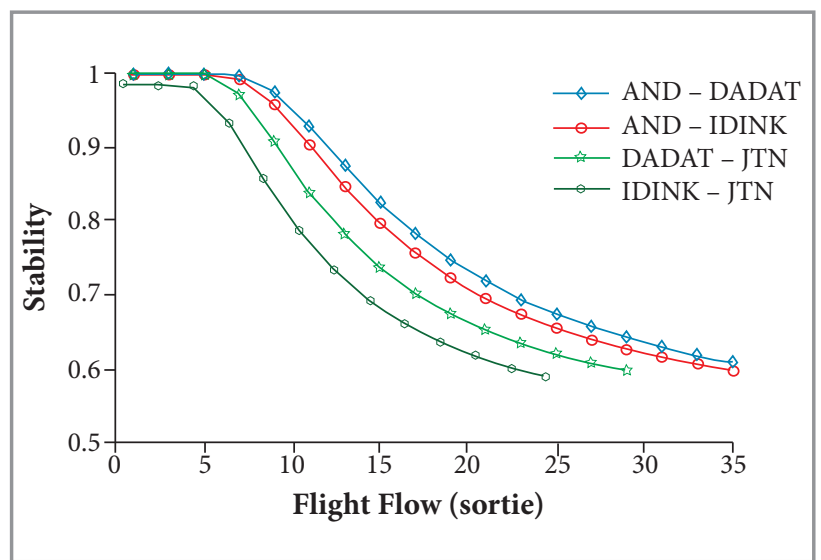

Figure 3. Route segment operation stability based on flight demand. network system becomes unstable, resulting in less efficiency of route network. Operating efficiency decreased significantly when the average workload intensity got close to $60 \%$ of the statistics time, which is consistent with the Doratask method that is adopted by UK logistics and the Flight Council. It also verifies the feasibility of the model.

In addition, due to the decrease of operation efficiency of route network, the route has not been fully utilized and the resource of route has been wasted. So the operation efficiency reduces when the capacity of segment is larger when the air flow is given. Therefore, we can improve the operation efficiency of the whole route network by distributing flight flow and using route segment flexibility.

\section{THE NUMERICAL EXAMPLE BASED ON REAL-TIME DATA}

We select the flight data in 19 time periods from 6:00 AM to 1:00 AM (each period is one hour). The numerical example based on real-time data is analyzed through the assessment model. Number 1 represents the period of 6:00 AM 7:00 AM; number 2 represents 7:00 AM 8:00 AM; number 19 represents the second day, 0:00 AM 1:00 AM; the rest can be calculated in the same manner. Figures 5 and 6, respectively, show the operation stability and the operation efficiency of the route network in the terminal area based on real-time data.

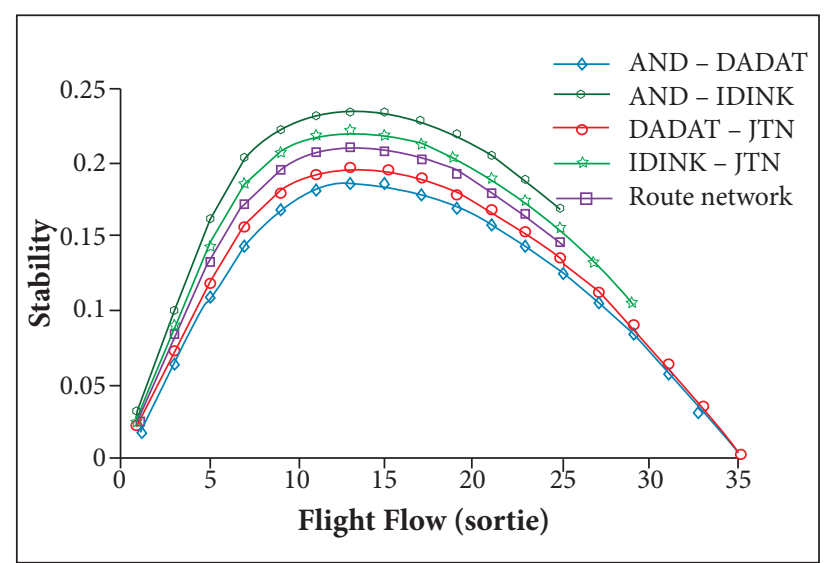

Figure 4. Operation efficiency of the route network based on flight demand.

Table 1. Original data of route segment.

\begin{tabular}{|c|c|c|c|c|}
\hline Route segment & AND - DADAT & AND - IDINK & DADAT - JTN & IDINK - JTN \\
\hline Length $[\mathrm{km}]$ & 22 & 33 & 75 & 76 \\
\hline Velocity $[\mathrm{km} / \mathrm{h}]$ & 540 & 360 & 450 & 400 \\
\hline Capacity/sortie & 38.00 & 26.00 & 35.00 & 29.00 \\
\hline
\end{tabular}


From Fig. 5, we know that the flying is unobstructed and operation stability is higher around 6:00 AM in the morning, because the airports and routes are just open. With the time continuing, the route network operation stability decreased gradually. In the first peak period of flight flow, the operation stability reached the first valley value around 10:00 AM and slowly recovered after 10:00 AM. Starting from 13:00 PM, the route network traffic becomes busy or congested and route network running stability is reduced to a lower level along with the increase of flight flow. From 05:00 PM to 10:00 PM, the air traffic flow is intensive in the route network and route network operation is relatively stable in this period. After 11:00 $\mathrm{PM}$, air traffic in terminal area is becoming unimpeded and the route network operation stability is higher.

Through this analysis, it can be seen that the operation stability of the selected route network in different times of a day is not identical with the changes of flight flow. The fluctuation of operation stability is very obvious.

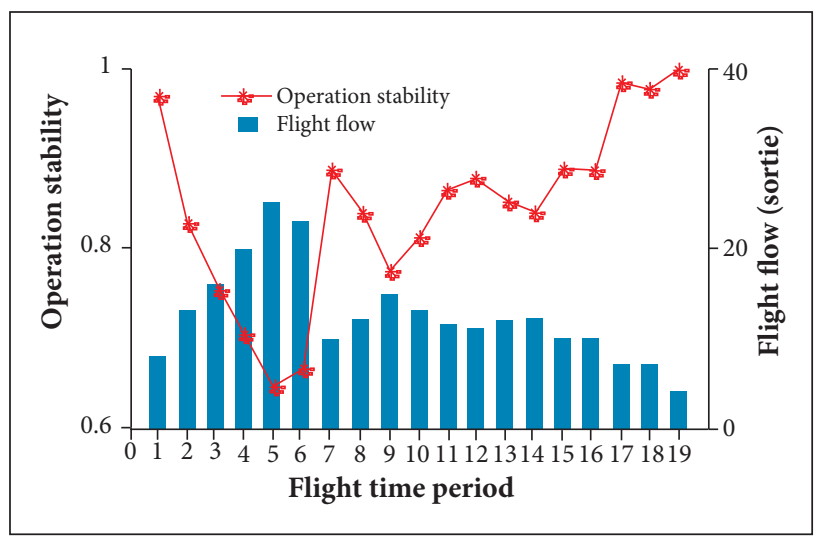

Figure 5. Operation stability of the route network based on real-time flight flow.

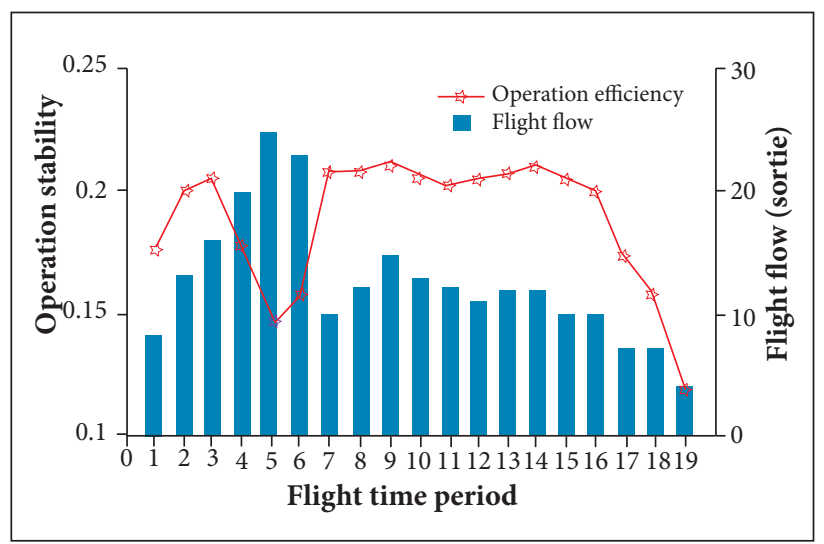

Figure 6. Operation efficiency of the route network based on real-time flight flow.
From Fig. 6, we can know that, during the time when airports and the routes begin to open at 9:00 AM, the efficiency of the route network in the terminal area increases along with the increase of flight flow. It can be known that resource in the terminal area is fully used and controller's workload keeps in a controllable scope. Additionally, the whole route network runs smoothly. The operation efficiency of terminal area route network gradually achieves the first trough at 10:00 AM. During the period, the controller's workload is larger and the route is in a congested state. As a result, service ability of route network in the terminal area is weak and the operation efficiency is also low. The operating efficiency begin to rebound with the increasing traffic flow after 11:00 AM.

The efficiency of route network in the terminal area is the highest around 02:00 PM. This suggests that route source is fully utilized, workload is quite suitable for controller and service ability in the terminal area is strong during this time. In other words, human ability and material resources are better used and it achieves a relatively input optimization. From 05:00 PM to 09:00 PM, the air traffic flow is appropriately intensive. So the operation efficiency level of this period is high and service ability of route network operation is fully brought into play. The efficiency is low after 11:00 PM, and the reason is that there is waste in human and material resources with the significant reduction in flight traffic.

Through the analysis, it can be drawn that the results of the terminal area route network efficiency obtained by using the assessment model are consistent with reality. So the model can be used as the assessment method for service ability and the operation state of route network. And it provides reference for resource allocation and organization optimization in the terminal area.

\section{CONCLUSION}

To describe the effect of the resource usage, controller's workload and air traffic flow stability on the running status of terminal area under different flight flows, the paper proposed the concept of terminal area route network operation efficiency. Firstly, according to the graph theory, a terminal area route network system chart can be constructed. Taking the flight flow as the starting point, the calculation parameters of "route segment traffic efficiency", "workload influence extent" and "route segment operation stability" are proposed by analyzing 
the influence factors of the terminal area route network operation efficiency. With comprehensive weighted method, an assessment model for the operation efficiency of terminal area route network is built based on flight demand and the three aspects already mentioned. Finally, it is applied to a given terminal area to verify the feasibility and practicability of the model.

This paper analyzes the influence factors of terminal area route network operation efficiency and establishes the calculation index of "route segment traffic efficiency", "workload influence degree" and "route segment operation stability". An assessment model on the operation efficiency of terminal area route network based on flight demand is built. Through the case analysis, the feasibility of the assessment model is verified. We can get that the route network operation stability fluctuates with the change of air traffic flow, and operating efficiency increases first and then decreases with the increase of air traffic flow. The model can be used to assess not only the operation efficiency of a single route segment in the terminal area, but also the real-time efficiency of the terminal area route network. And the model will benefit the airspace management and serve as a theoretical basis for scientific exploration of airspace system. Additionally, the model can provide reference for the dynamic distribution of traffic, according to the different operation efficiencies.

This article proposed an operation efficiency assessment model of route network in terminal area based on flight traffic and did not considered in detail the changes in controller's workload, different airspace structures and operating environment. Future studies will be to control the workload and the external environment changes in the specific analysis and calculations, to establish a more precise terminal area route network efficiency evaluation model. Additionally, the next study will provide the regression analysis of fight flow and efficiency to distribute the air traffic flow dynamically by the model.

\section{ACKNOWLEDGEMENTS}

This study was supported by the National Natural Science Foundation Project of China (71171190) and the 2010 Fundamental Research Funds for the Central Universities (2XH2011B004).

\section{REFERENCES}

Asakura Y (1996) Reliability measures of an origin and destination pair in a deteriorated road network with variable flows. In: Bell MGH. Transportation networks: recent methodological advances. Oxford: Pergamon Press. p. 273-287.

Bloem M, Kopardekar P (2008) Combining airspace sectors for the efficient use of air traffic control resources. Proceedings of the AIAA Guidance, Navigation, and Control Conference and Exhibit; Honolulu, Hawaii.

D'Ariano A, Pacciarelli D, Pistelli M, Pranzo M (2015) Real-time scheduling of aircraft arrivals and departures in a terminal maneuvering area. Networks 65(3):212-227. doi: 10.1002/net.21599

D'Ariano A, Pistelli M, Pacciarelli D (2012) Aircraft retiming and rerouting in vicinity of airports. IET Intelligent Transport Systems 6(4):433-443. doi: 10.1049/iet-its.2011.0182

Federal Aviation Administration (1997) National airspace system performance measurement overview. Virginia: Center for Advanced Aviation System Development.

Hou L, Jiang F (2000) Study on the reliability of urban road network. Systems Engineering 18(5):44-48.

Jenelius E, Petersen T, Mattsson LG (2006) Importance and exposure in road network vulnerability analysis. Transport Res A-Pol 40(7):537560. doi: 10.1016/j.tra.2005.11.003

Latora V, Marchiori M (2002) Is the Boston subway a small-world network? Physica A 314(1):109-113.
Latora V, Marchiori M (2004) How the science of complex networks can help developing strategies against terrorism. Chaos Soliton Fract 20(1):69-75. doi: 10.1016/s0960-0779(03)00429-6

Li X, Zhang Z, Hou R (2009a) Research of the structural stability of the route network system. Journal of Transportation Engineering and Information 7(1):80-84.

Li X, Zhang Z, Wang L (2009b) Terminal area capacity evaluation method based on following stability. Systems Engineering: Theory \& Practice 29(2):173-179.

Ming $G$ (2006) The application research on reliability of route travel time and its distribution in the aspect of traffic. Harbin: Harbin Institute of Technology.

Performance Review Unit [2001] Cost effectiveness and productivity KPls. Working Paper prepared by the KPI Drafting Group, Version 1.0, EUROCONTROL.

Qin J, Shi F (2008) A new measure for transportation network efficiency and its application. Systems Engineering 26(4):94-98.

Rakas J, Hansen M (2002) Methodology for estimating terminal airspace service availability and system effectiveness. AIAA's Aircraft Technology, Integration and Operations.

Samà M, D’Ariano A, D'Ariano P, Pacciarelli D (2014) Optimal aircraft scheduling and routing at a terminal control area during disturbances. Transport Res C-Emer 47(Pt 1):61-85. doi: 10.1016/j. trc.2014.08.005 
Samà M, D’Ariano A, Pacciarelli D (2013) Rolling horizon approach for aircraft scheduling in the terminal control area of busy airports. Procedia - Social and Behavioral Sciences 80:531-552. doi: 10.1016/j.sbspro.2013.05.029

Sheffi Y (1985) Urban transportation networks: equilibrium analysis with mathematical programming methods. Cambridge, MA: Massachusetts Institute of Technology.

Wang S, Huang W, Lu Z (2006) Deduction of link performance function and its regression analysis. Journal of Highway and Transportation Research and Development 23(4):107-110.

Watts DJ, Strogatz SH (1998) Collective dynamics of 'small-world' networks. Nature 393(6684):440-442. doi: 10.1038/30918

Wilson RJ (1986) An Eulerian trail through Königsberg. J Graph Theor
10(3):265-275. doi: 10.1002/jgt.3190100305

Xu G, Yu J (2003) An approach of route segment based collision alert technology. Computer Engineering and Applications 39(4):213-214.

Zhan ZH, Zhang J, Li Y, Liu O, Kwok SK, Ip WH, Kaynak O (2010) An efficient ant colony system based on receding horizon control for the aircraft arrival sequencing and scheduling problem. IEEE Trans Intell Transp Syst 11(2):399-412.

Zhang J, Hu M, Wu Z (2013) An improved integrated evaluation method on performance of air traffic control based on BP Network. Journal of Southwest Jiaotong University 48(3):553-558.

Zhu S, Wang W, Deng W (2000) Research on traffic network reliability and access road algorithm. China Journal of Highway and Transport 13(1):91-94. 